\title{
Nociceptive Behavior Induced by Chemotherapeutic Paclitaxel and Beneficial Role of Antioxidative Pathways
}

\author{
Hua MIAO ${ }^{1^{*}}$, Jinrong XU ${ }^{1 *}$, Dongsheng XU ${ }^{1}$, Xiaohui MA ${ }^{1}$, Xiaoming ZHAO ${ }^{1}$, Li LIU $^{1}$ \\ * These authors contributed equally to this work. \\ ${ }^{1}$ Tumor Center, The First Hospital of Jilin University, Changchun, Jilin, China
}

Received May 5, 2018

Accepted July 10, 2018

Epub Ahead of Print October 23, 2018

\section{Summary}

Paclitaxel is used for the treatment of several types of cancers. However, one of the significant limiting complications of paclitaxel is painful peripheral neuropathy during its therapy. In this study we examined the engagement of antioxidative signal pathway of the dorsal root ganglion (DRG) in mechanical and thermal hypersensitivity evoked by paclitaxel. Behavioral test was performed to determine mechanical and thermal sensitivity in rats. Western blot analysis and ELISA were used to examine expression of Nrf2-antioxidant response element (ARE) and superoxide dismutases (SOD); and the levels of products of oxidative stress in the DRG. Our results show that paclitaxel increased mechanical and thermal sensitivity as compared with vehicle control animals. Paclitaxel also impaired Nrf2-ARE and SOD in the DRG and amplified products of oxidative stress, namely 8-isoprostaglandin $\mathrm{F} 2 \mathrm{a}$ and 8-hydroxy-2'deoxyguanosine. Systemic administration of SOD mimetic using tempol, antioxidant vitamin $\mathrm{C}$ or blocking oxidative pathway using NADPH oxidase inhibitor (GKT137831) attenuated mechanical and thermal hypersensitivity induced by paclitaxel. This inhibitory effect was accompanied with decreases of proinflammatory cytokines (PICs) such as IL-1 $\beta$, IL- 6 and TNF-a in the DRG. In conclusion, the data revealed impairment of Nrf2-ARE and heightened oxidative and PIC signals in the DRG of paclitaxel rats, leading to neuropathic pain. Balancing of reactive oxygen species by supplying antioxidants and/or inhibiting NADPH oxidase appears significant to yield beneficial effects in neuropathic pain conditions after chemotherapeutic paclitaxel.

\section{Key words}

Paclitaxel • Neuropathic pain • Nrf2-ARE • SOD • NOX • Pro-inflammation

\section{Corresponding authors}

X. Zhao and L. Liu, Tumor Center, The First Hospital of Jilin University, 71 Xinmin Street, Changchun, Jilin 130021, China. E-mail: dxliu@yahoo.com

\section{Introduction}

One of the most common and distressing symptoms suffered by patients with progression of cancer is pain (Hanna et al. 2013). Cancer pain mainly arises from a tumor compressing or infiltrating tissue; from nerve and other changes caused by a hormone imbalance or immune response; and/or from treatments and diagnostic procedures (Pasetto et al. 2006, Hanna et al. 2013). It should be noted that chemotherapy and radiotherapy produce painful conditions persisting long after treatment has ended (Hoskin 2008, Portenoy 2011, Hanna et al. 2013). As a result, how to effectively manage cancer pain related to these therapies becomes am important issue for treatment and management of cancer patients in clinics.

Paclitaxel is a widely prescribed chemotherapeutic agent for the treatment of breast, lung and other cancers, but causes a variety of serious side effects, including peripheral neuropathy, leukopenia, joint or muscle pain, vomiting and alopecia (Ghersi et al. 2015). Chemotherapy-induced peripheral neuropathy causes severe sensory disturbances that range from mild tingling to spontaneous painful burning paresthesia affecting the sensory nerves to the hands and feet (Dougherty et al. 2004) and can persist long after treatment cessation (Tanabe et al. 2013) in up to $68 \%$ of chemotherapy cancer patients (Seretny et al. 2014). As traditional 
analgesics generally lack efficacy in treating this condition (Kim et al. 2015), a pressing need exists for novel analgesic strategies. In another word, treatment options for these abnormal sensations have been restricted, partly due to a poor understanding of the underlying mechanisms responsible for neuropathic pain induced by chemotherapeutic paclitaxel.

A number of studies have indicated that oxidative stress induced by an imbalance in redox homeostasis is critically involved in the induction of neuropathic pain (Jaggi and Singh 2011, KallenbornGerhardt et al. 2013). Oxidative stress occurs due to overproduction of free radicals, i.e. reactive oxygen species (ROS) such as superoxide anion $\left(\mathrm{O}_{2}^{-}\right)$or hydrogen peroxide $\left(\mathrm{H}_{2} \mathrm{O}_{2}\right)$ together with a failure of antioxidant defense mechanisms (Altenhofer et al. 2012). Intracellular accumulation of ROS and disturbances in the cellular redox state are the consequences that then induce not only to general oxidative stress responses like DNA fragmentation, breakdown of signaling pathways, and tissue damage, but also neuronal degeneration and sensitization in pain conditions (Salvemini et al. 2011). Prevention of ROS generation, rather than scavenging already formed free radicals, may represent another possibility to target oxidative stress (Altenhofer et al. 2015). Accordingly, balancing of ROS by administration of antioxidants or free radical scavengers and inhibition of ROS generation is necessary to play a beneficial role in modulating neuropathic pain conditions.

Nuclear factor (erythroid-derived 2)-like 2 (Nrf2) is a transcription factor and as a basic leucine zipper protein it regulates the expression of antioxidant proteins protecting against oxidative damage triggered by injury and inflammation (Moi et al. 1994). Numerous drugs that stimulate the Nrf2 pathway were used for treatment of diseases caused by oxidative stress (Gold et al. 2012). Thus, in this study, in order to determine the engagement of antioxidative signal in paclitaxel-induced neuropathic pain we first examined Nrf2-antioxidant response element (ARE) pathway in the dorsal root ganglion (DRG) of control rats and rats with injection paclitaxel. We further examined superoxide dismutases (SOD), a class of enzymes to catalyze the dismutation of superoxide; 8-isoprostaglandin F2 $\alpha$ (8-iso PGF2 $\alpha$, a product of oxidative stress); and 8-hydroxy-2'deoxyguanosine $(8-\mathrm{OHdG}$, a key biomarker of protein oxidation). We hypothesized that paclitaxel impairs Nrf2 and Nrf2-regulated NADPH quinone oxidoreductase-1 (NQO1), attenuates the levels of SOD and amplifies oxidative production in the DRG of rats. This process subsequently increases pro-inflammatory cytokines (PICs), namely IL-1 $\beta$, IL-6 and TNF- $\alpha$ in the DRG, thereby resulting in mechanical and thermal hypersensitivity. We further hypothesized that supplying SOD or inhibiting oxidative NADPH oxidase (NOX) attenuates the amplified levels of PICs and alleviates paclitaxel-evoked mechanical and thermal pain.

\section{Methods}

\section{Animals}

All animal protocols were in accordance with the guidelines of the International Association for the Study of Pain and approved by the Institutional Animal Care and Use Committee of Jilin University. Adult male Sprague-Dawley rats $(200-250 \mathrm{~g})$ were housed in individual cages with free access to food and water and were kept in a temperature-controlled room $\left(25^{\circ} \mathrm{C}\right)$ on a 12/12 h light/dark cycle.

\section{A model of neuropathic pain and administration of drugs}

Paclitaxel (Tocris Biosci) was dissolved in a $40 \%$ dimethyl sulfoxide (DMSO) (Chen et al. 2011). Neurotoxicity was induced in rats by intraperitoneal (i.p.) injection of paclitaxel $(1 \mathrm{mg} / \mathrm{kg} /$ one injection in $0.5 \mathrm{ml}$ of volume) every two days and a total of four injections were given. Control rats received four injections of $40 \%$ DMSO $(0.5 \mathrm{ml})$ every two days. Mechanical and thermal sensitivity was tested after paclitaxel injection. Mechanical and thermal hypersensitivity were fully developed after four injections of paclitaxel and then six hours of behavioral test were performed (as shown in Fig. 1). In separate rats, DRG tissues were removed for western blot analysis and ELISA measurement of 8-iso PGF $2 \alpha$ and $8-O H d G$.

Prior to each of experiments, $0.5 \mathrm{ml}$ of dimethyl sulfoxide (DMSO) as vehicle control, a mimetic of SOD (tempol, $20 \mathrm{mg} / \mathrm{kg}$; Sigma-Aldrich, USA); vitamin C $(500 \mathrm{mg} / \mathrm{kg}$; Sigma-Aldrich, USA) and inhibitor of NOX4 (GKT137831, 1 mg/kg; Cayman Chemical, USA) were i.p. given (daily for 5 days; as shown in Fig. 1). The volume of injected drugs was $0.5 \mathrm{ml}$. In a subset of experiments, in order to examine the effects of oxidative stress pathways on PICs tempol, vitamin C and GKT137831were i.p. given (daily for 5 days; as shown in Fig. 1), respectively. Then, rats were euthanized by sodium pentobarbital and DRG tissues were removed for ELISA measurements of IL-1 $\beta$, IL- 6 and TNF- $\alpha$. 


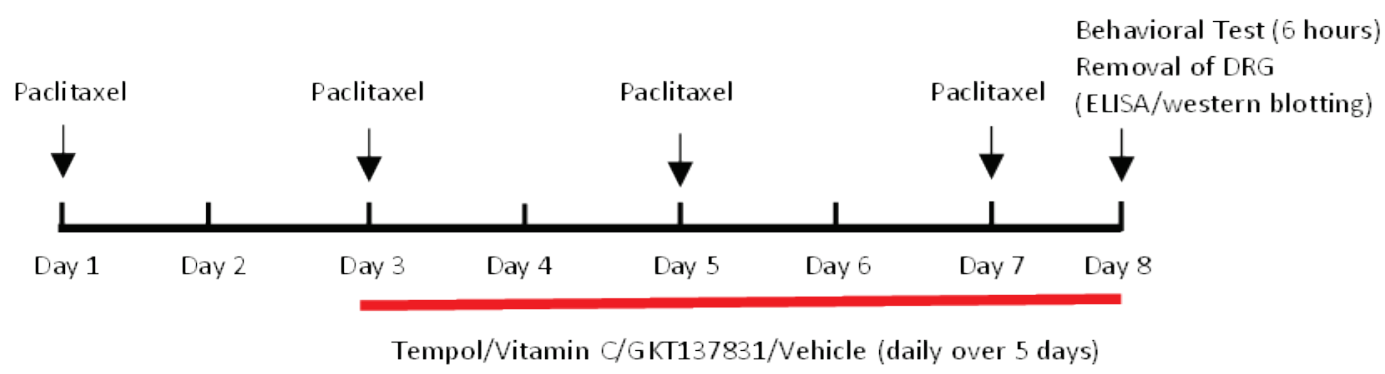

Fig. 1. A schematic diagram showing the schedule giving drugs. Paclitaxel was injected [intraperitoneally (i.p.), $1 \mathrm{mg} / \mathrm{kg}$ body weight every other day] (indicated as arrows). An equivalent volume of vehicle was given in control animals. Then, tempol, vitamin C, GKT137831 and vehicle were administered (indicated as red bar) daily for 5 days. On the $8^{\text {th }}$ day, behavioral tests were performed and in the separate rats the DRGs were removed for ELISA measurements and western blot analysis.

\section{Behavioral test}

To quantify the mechanical sensitivity of the hind paw, rats were placed in individual plastic boxes and allowed to acclimate for $>30 \mathrm{~min}$. Mechanical paw withdrawal threshold (PWT) of rat hind paw in response to the stimulation of von Frey filaments was determined. A series of calibrated von Frey filaments (ranging from 0.5 to $18.0 \mathrm{~g}$ ) were applied perpendicularly to the plantar surface of the hind paw with a sufficient force to bend the filaments or until paw withdrew. In the presence of a response, the filament of next lower force was applied. In the absence of a response, the filament of next greater force was applied. To avoid injury during tests, the cutoff strength of the von Frey filament was $18 \mathrm{~g}$. The tactile stimulus producing a $50 \%$ likelihood of withdrawal was determined using the "up-down" method (Chaplan et al. 1994). Each trial was repeated 2 times at approximately 2 min intervals. The mean value was used as the force produced a withdrawal response.

To determine thermal hyperalgesia, rat paw withdrawal latency (PWL) to a radiant heat was measured. Rats were placed individually in plastic cages on an elevated glass platform and allowed for $30 \mathrm{~min}$ acclimation. Each hind paw received three stimuli with a 10 min interval, and the mean of the three withdrawal latencies was defined as PWL. The heat was maintained at a constant intensity. To prevent tissue damage, the cut-off latency was set at $20 \mathrm{~s}$. All the behavioral tests were performed in a blind style.

\section{ELISA measurements}

The levels of 8-iso PGF $2 \alpha$ and 8 -OHdG in the DRGs (L4-L6) were examined using an ELISA assay kits (obtained from Promega Co. and Abcam Co.) according to the provided description and modification. Briefly, polystyrene 96-well microtitel immunoplates were coated with affinity-purified rabbit primary antibodies. Parallel wells were coated with purified rabbit IgG for evaluation of nonspecific signal. After overnight incubation, plates were washed. Then, the diluted samples and 8-iso PGF2 $\alpha / 8-O H d G$ standard solutions (100 pg/ml$100 \mathrm{ng} / \mathrm{ml}$ ) were distributed in each plate. The plates were washed and incubated with anti-8-iso PGF2 $\alpha / 8$ OHdG galactosidase. Then, the plates were washed and incubated with substrate solution. After incubation, the optical density was measured using an ELISA reader. Using the same method, the levels of PICs including IL-1 $\beta$, IL-6 and TNF- $\alpha$ were examined using an ELISA assay kit (Promega Co.).

\section{Western blot analysis}

Briefly, DRGs (L4-L6) were removed and total protein of DRG tissues was then extracted by homogenizing sample in ice-cold immunoprecipitation assay buffer. The lysates were centrifuged and the supernatants were collected. After being denatured by heating at $95{ }^{\circ} \mathrm{C}$ in an SDS sample buffer, the supernatant samples were loaded onto Mini-Protean TGX Precast gels and electrically transferred to a polyvinylidene fluoride membrane. The membrane was then incubated overnight with diluted primary antibodies: rabbit anti-Nrf2 (at 1:250), anti-NQO1 (at 1:500), anti-SOD (at 1:500) and anti-NOX4 (at 1:250). After being washed, the membrane was incubated with horseradish peroxidaselinked anti-rabbit secondary antibody (diluted at 1:500) and visualized for immunoreactivity. All antibodies were obtained from Abcam Co. (USA) and Santa Cruz Biotech (USA). The membrane was stripped and incubated with anti- $\beta$-actin to show equal loading of the protein. The bands recognized by the primary antibody were visualized by exposure of the membrane onto an X-ray film. Then, the film was scanned and the optical densities of primary antibodies and $\beta$-actin bands were determined using the Scion Software. Values for densities of 
immunoreactive bands/ $\beta$-actin band from the same lane were determined. Each of the values was then normalized to a control sample.

\section{Statistical analysis}

All data were analyzed using a one-way repeated-measures analysis of variance for different time courses between two experimental groups. Values were presented as means \pm standard deviation of mean. For all analyses, differences were considered significant at $P<0.05$. All statistical analyses were performed by using SPSS for Windows version 13.0 (SPSS Inc., Chicago, IL, USA).

A
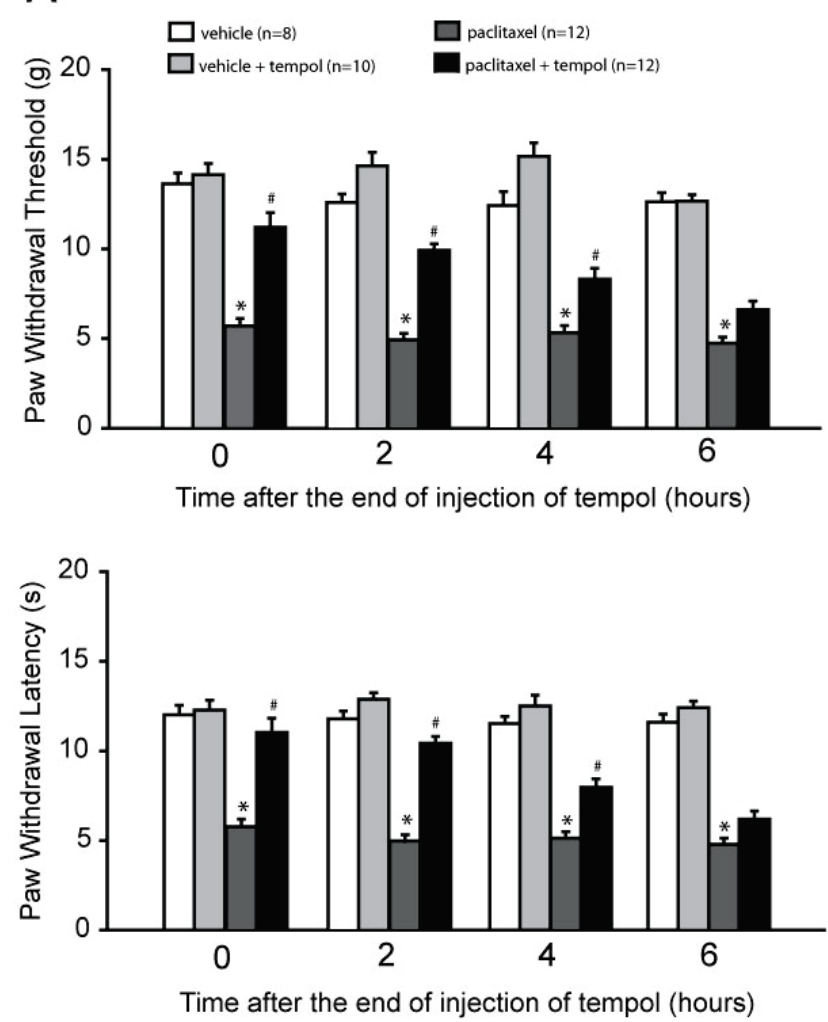

\section{Results}

\section{Mechanical and thermal sensitivity}

Systemic injection of paclitaxel significantly decreased PWT as compared with vehicle injection $(P<0.05$, paclitaxel rats $/ \mathrm{n}=12$ vs. vehicle control rats $/ \mathrm{n}=8$ ). Figure $2 \mathrm{~A}$ (top panel) demonstrates that PWT was increased after i.p. injection of tempol in paclitaxel rats ( $\mathrm{n}=12 ; P<0.05$ vs. paclitaxel rats with no tempol). As tempol was given in paclitaxel rats, the inhibitory effects on mechanical hypersensitivity lasted for several hours after the end of its administration. In addition, the increase of PWT evoked by tempol was minimal in control rats ( $\mathrm{n}=10 ; P>0.05$ vs. vehicle control rats).

B
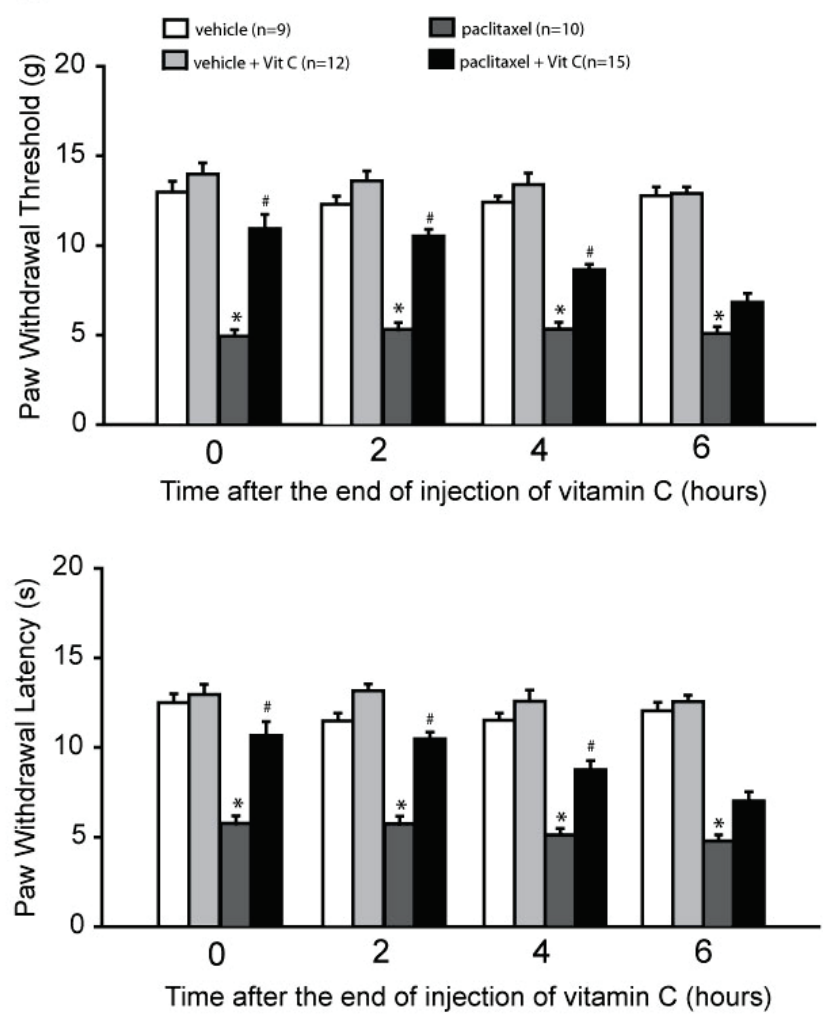

Fig. 2. Mechanical and thermal sensitivity in control rats and paclitaxel rats after application antioxidants. (A), top panel: Effects of administration of tempol on mechanical sensitivity in vehicle control rats and paclitaxel rats. As compared with vehicle injection, tempol increased PWT in paclitaxel rats. Bottom panel: Effects of tempol on thermal sensitivity in control rats and paclitaxel rats. Tempol increased PWL in paclitaxel rats. Note that the increases of PWT and PWL evoked by tempol were insignificant in control rats. * $P<0.05$ vs. vehicle control. ${ }^{\#} P<0.05$ vs. paclitaxel rats with no treatment. The number of animals is indicated on the figure. (B), top panel: Effects of administration of vitamin C on PWT on mechanical sensitivity in control rats and paclitaxel rats. Vitamin C increased PWT in paclitaxel rats as compared with vehicle injection, but the amplitude of PWT increases evoked by vitamin C was minimal in control rats. Bottom panel: Effects of vitamin $C$ on thermal sensitivity in control rats and paclitaxel rats. Vitamin C elevated PWL in paclitaxel rats, but not in control rats. ${ }^{*} P<0.05$ vs. vehicle control. ${ }^{\#} P<0.05$ vs. paclitaxel rats with no treatment. The number of animals is indicated on the figure.

Likewise, paclitaxel injection also significantly diminished PWL as compared with vehicle injection. Figure 2A (bottom panel) further shows that tempol significantly attenuated thermal hypersensitivity in paclitaxel rats $(P<0.05$, paclitaxel rats $/ \mathrm{n}=12$ vs. paclitaxel rats with tempol $/ \mathrm{n}=12)$. The effects of injection of tempol 
on PWL were insignificant in control rats $(P>0.05$, vehicle control rats $/ \mathrm{n}=8$ vs. vehicle control rats with tempol/n=10).

In addition, the effects of vitamin $\mathrm{C}$ on mechanical and thermal sensitivity were examined. Figure 2B demonstrates that vitamin $\mathrm{C}$ increased PWT and PWL in paclitaxel rats $(n=15)(P<0.05$ vs. paclitaxel rats without vitamin $C ; n=10)$, but the effects were not significant in control rats $(P>0.05$, paclitaxel $/ \mathrm{n}=12$ vs. vehicle/n=9). The time course of vitamin $C$ was similar to the one observed as tempol was applied.

We also examined the role played by NOX inhibition in mediating mechanical and thermal hypersensitivity in paclitaxel rats. Figure 3 (top panel) shows that blocking NOX4 using GKT137831 attenuated mechanical hypersensitivity in paclitaxel rats as compared with vehicle injection $(P<0.05$, paclitaxel rats $/ \mathrm{n}=12$ vs. paclitaxel rats with GKT137831/n=15). The inhibitory effects of GKT137831 were observed to last for several hours after the end of its administration. The effects of GKT137831 were insignificant in control rats $(P>0.05$, vehicle control rats $/ \mathrm{n}=8$ vs. vehicle control rats with GKT137831/n=12). In addition, Figure 3 (bottom panel) demonstrates that inhibition of NOX4 using GKT137831 alleviated thermal hypersensitivity in paclitaxel rats, but not in control rats.
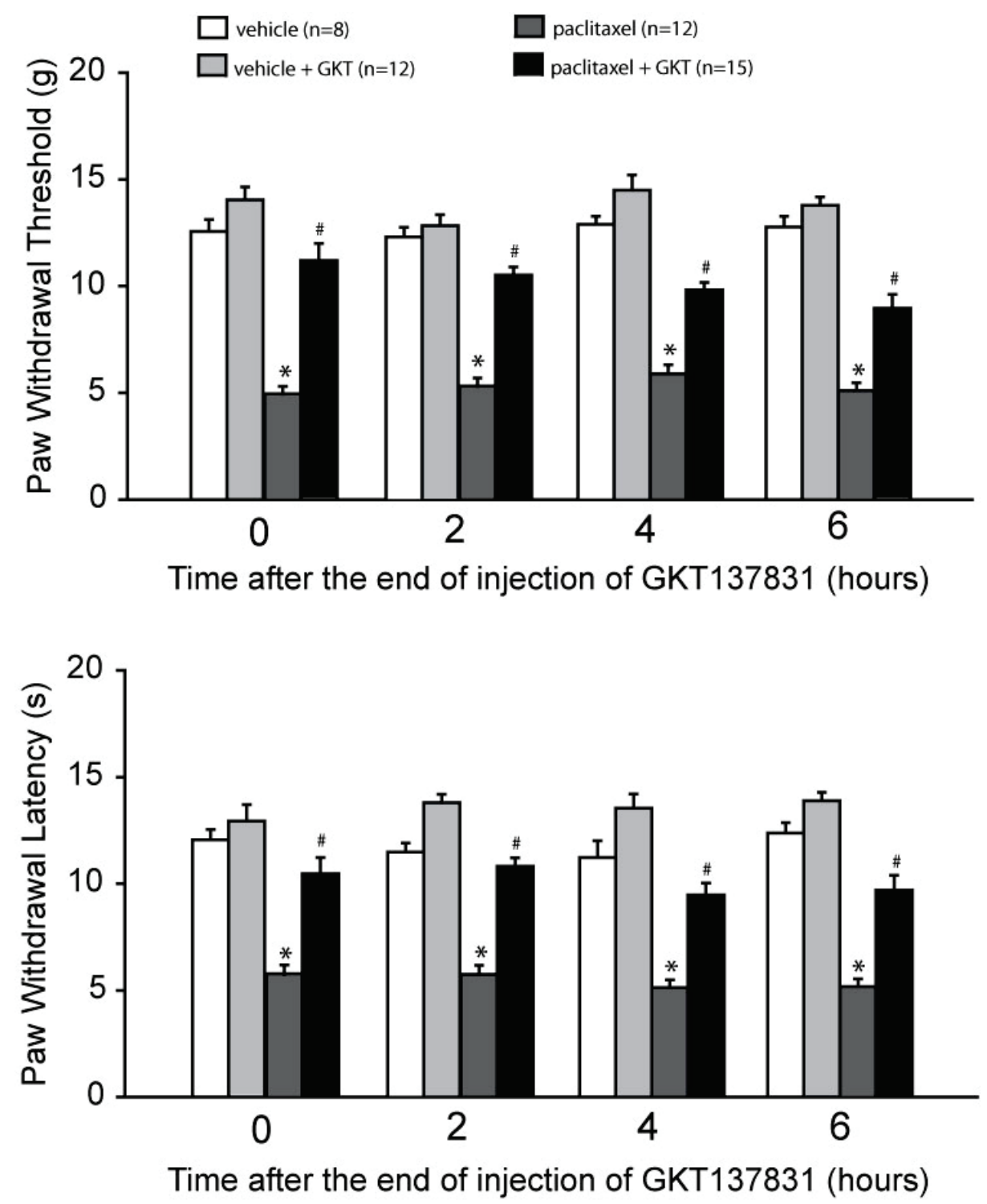

Fig. 3. Mechanical and thermal sensitivity in control rats and paclitaxel rats after inhibition of NOX4. Top panel: As compared with vehicle injection, GKT137831 significantly increased PWT in paclitaxel rats. Bottom panel: GKT137831 increased PWL in paclitaxel rats. Note that the increases of PWT and PWL evoked by GKT137831 were insignificant in control rats. ${ }^{*} P<0.05$ vs. vehicle control. ${ }^{\#} P<0.05$ vs. paclitaxel rats with no treatment. The number of animals is indicated on the figure. 
Expression of Nrf2-ARE and oxidative stress signal pathways

We first examine the protein levels of $\mathrm{Nrf} 2$ and Nrf2-regulated NQO1 in the DRG. Figure 4A shows that the protein expression levels of $\mathrm{Nrf} 2$ and Nrf2-regulated NQO1 were decreased in paclitaxel rats $(P<0.05$, paclitaxel rats vs. control rats; $n=6-8)$. In the similar way, Figure 4B further shows that paclitaxel led to downregulation of SOD expression $(P<0.05$, paclitaxel rats vs. controls rats; $n=6-8)$. In contrast, NOX4 was amplified by paclitaxel as shown in Figure 4C $(P<0.05$, paclitaxel rats vs. controls rats; $n=6$ in each group). In addition, we examined products of oxidative stress in the DRG of control rats and paclitaxel rats as shown in Figure 4D. 8-iso PGF2 $\alpha$ and $8-\mathrm{OHdG}$ were significantly increased in the DRG of paclitaxel rats $(P<0.05$ vs. control rats, $n=15$ in each group). Note that all samples were obtained from individual rats in those experiments using ELISA measurement and western blot analysis.

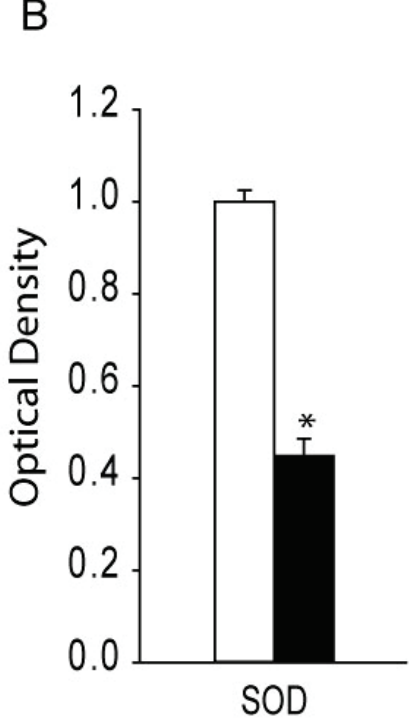

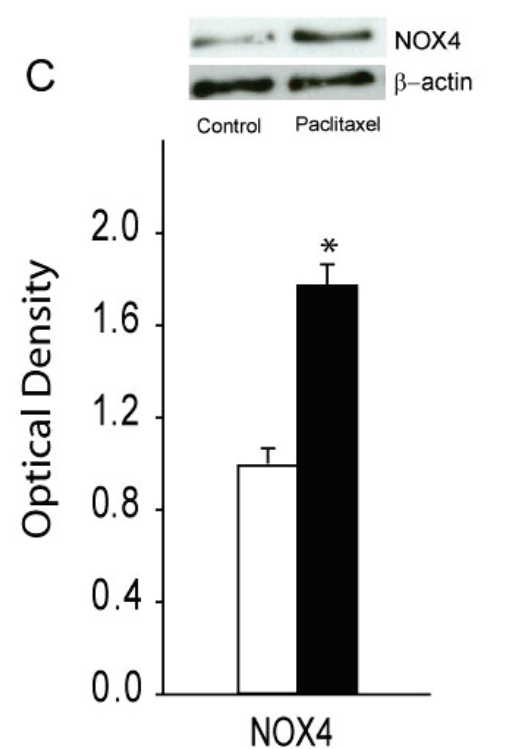
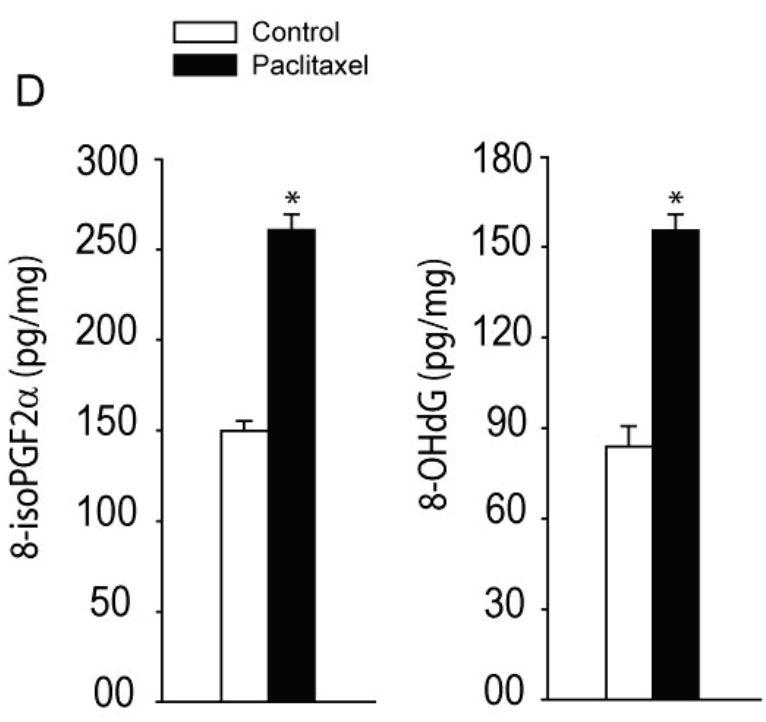

Fig. 4. Effects of paclitaxel on signal pathways of oxidative stress. (A, B), Protein expression levels of Nrf2-ARE and SOD. Top panel and bottom panel are representative bands and averaged data: Nrf2/NQO1 and SOD were decreased in the DRG of paclitaxel rats. $* P<0.05$, paclitaxel rats vs. control. $\mathrm{n}=6-8$ in each group. (C), Representative bands and averaged data, showing that protein expression levels of NOX4 were amplified by paclitaxel. $* P<0.05$, paclitaxel rats vs. control. $\mathrm{n}=6$ in each group. (D), Systemic administration of paclitaxel amplified the levels of oxidative products 8-iso PGF2a and 8-OHdG in the DRG. $* P<0.05$ vs. control rats. $n=15$ in each group. " $n$ " represents individual rat and all samples were obtained from different rats in those experiments. 


\section{Levels of PICs}

In additional experiments, we examined the effects of paclitaxel on the levels of PICs including IL-1 $\beta$, IL- 6 and TNF- $\alpha$ in the DRG. Figure 5 shows that IL-1 $\beta$, IL- 6 and TNF- $\alpha$ were significantly increased in paclitaxel rats $(\mathrm{n}=12)$ as compared with vehicle control rats $(n=8)$. Furthermore, administration of individual tempol ( $\mathrm{n}=15)$, vitamin $\mathrm{C}(\mathrm{n}=16)$ and GKT137831 $(\mathrm{n}=15)$ significantly attenuated amplifications of PICs evoked by paclitaxel. It is noted that no significant differences were observed in the inhibitory effect of tempol, vitamin $\mathrm{C}$ and GKT137831 on the levels of IL-1 $\beta$, IL- 6 and TNF- $\alpha$ in the DRG of paclitaxel rats.
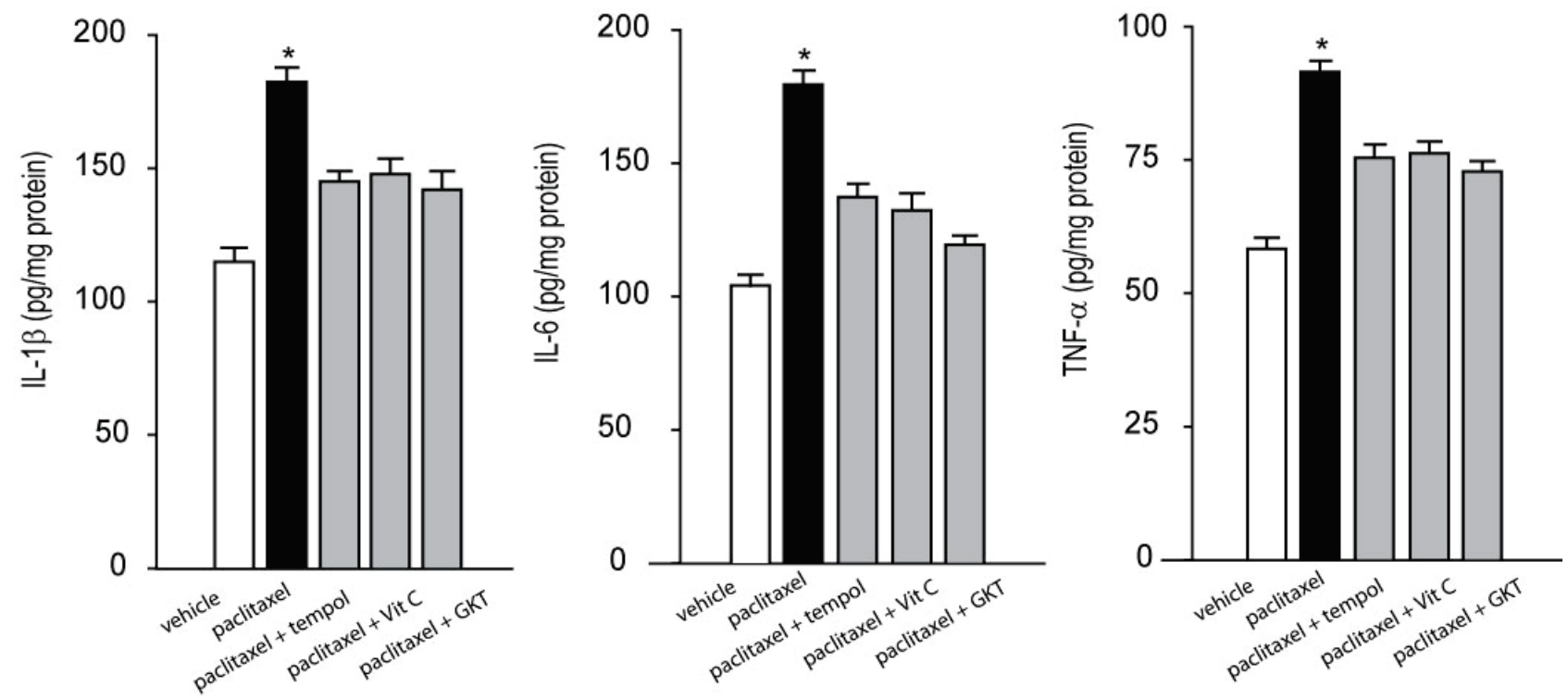

Fig. 5. Levels of PICs in control rat and paclitaxel rats. Paclitaxel increased IL-1 $\beta$, IL- 6 and TNF- $a$ as compared with vehicle control rats. Systemic administration of tempol, vitamin C and GKT137831 significantly attenuated amplifications of PICs evoked by paclitaxel. $* P<0.05$ vs. control rats and paclitaxel rats with tempol, vitamin $\mathrm{C}$ and GKT137831. $\mathrm{n}=8$ in vehicle control; $\mathrm{n}=12$ in paclitaxel; $\mathrm{n}=15$ in paclitaxel + tempol; $n=16$ in paclitaxel + vitamin $C$ and $n=15$ in paclitaxel + GKT137831.

\section{Discussion}

Prior studies have shown that injection of paclitaxel produces neuropathic pain in rats including mechanical hyperalgesia and thermal hypersensitivity several days after initiation of the chemotherapy regimen in rats (Chen et al. 2011). The signs of mechanical hyperalgesia and thermal hypersensitivity lasted for several weeks after administration of paclitaxel (Chen et al. 2011). Thus, in this study we employed this well-established rat model to examine the mechanisms leading to neuropathic pain induced by paclitaxel. Using the same intervention, in our current study we observed that PWT declined to evoke mechanical withdrawal and PWL decreased to induce thermal withdrawal after injection of paclitaxel. This is consistent with the previous findings (Chen et al. 2011). Results of the current study also demonstrated that the expression of Nrf2-ARE signal and SOD expression was downregulated in the DRG of paclitaxel rats as compared with control rats. The expression of NOX4, the levels of oxidative products 8 -iso PGF $2 \alpha / 8-\mathrm{OHdG}$ and PICs such as IL-1 $\beta$, IL- 6 and TNF- $\alpha$ were amplified in the DRG of paclitaxel rats. Our study further demonstrated that PICs were decreased and mechanical and thermal hypersensitivity were attenuated in paclitaxel rats after a NOX4 inhibitor GKT 137831, SOD mimetic tempol or antioxidant vitamin $\mathrm{C}$ was systemically injected.

The role of oxidative stress in chemotherapyinduced peripheral neuropathy has been studied. As a non-specific ROS scavenger, N-tert-butyl- $\alpha$ phenylnitrone $(\mathrm{PBN})$ was reported to inhibit the development of paclitaxel-induced mechanical hypersensitivity (Fidan-boylu et al. 2011). Also, tempol inhibited the development and maintenance of paclitaxelinduced mechanical hypersensitivity, but was ineffective on cold allodynia (Fidanboylu et al. 2011, Kim et al. 2017). In addition, the activity of different antioxidant enzymes in the DRG and peripheral sensory nerves were examined during the time course of paclitaxel-induced 
painful neuropathy. Enhanced activity of mitochondrial and cellular endogenous antioxidant enzymes in the DRG and peripheral nerves was observed, however this was inadequate and delayed in its onset leading to excessive ROS in peripheral sensory axons (Duggett et al. 2016). Others have demonstrated an impaired mitochondrial antioxidant response after paclitaxel (Janes et al. 2013). In our current study, we demonstrated that expression of SOD was decreased in the DRG of paclitaxel rats and tempol/vitamin $\mathrm{C}$ attenuated paclitaxel-induced mechanical and thermal hypersensitivity. Collectively, these data suggest that mitochondrial ROS is causal to the development and maintenance of paclitaxel-induced peripheral neuropathy.

In addition to antioxidants and/or free radical scavengers, it is also interesting to determine the effects of decreasing ROS generation on paclitaxel-induced mechanical and thermal hypersensitivity. One source of ROS production is the enzyme family of NOX. The rodent genome encodes four genes that contain the catalytic NOX subunit, namely NOX1, NOX2, NOX3, and NOX4 (Altenhofer et al. 2012). This electron-transferring subunit is constitutively inactive in resting cells and generates ROS only upon activation, e.g. after noxious stimuli (Salvemini et al. 2011). While NOX2 activation is predominantly associated with innate immunity mediated host defense and NOX1 with blood pressure control and related vascular mechanisms (Gavazzi et al. 2006, Lam et al. 2010), NOX4 was shown highly expressed under ischemic conditions in the central nervous system, e.g. ischemic stroke (Suzuki et al. 2012). Evidence has also identified activation of NOX4 as a causative factor that contributes to inflammatory or neuropathic pain role in the peripheral nervous system (Kallenborn-Gerhardt et al. 2012). Thus, in the current study we further determined expression levels of NOX4 in the DRG of paclitaxel rats. We observed that NOX4 was upregulated after administration of paclitaxel. Moreover, NOX4 inhibitor GKT137831 alleviated paclitaxel-induced mechanical and thermal hypersensitivity.

Furthermore, paclitaxel was reported to increase the level of IL-1 $\beta$, IL- 6 and TNF- $\alpha$ in the DRG of rats thereby inducing pain behaviors (Kim et al. 2016, Kim et al. 2017). Several molecular mediators in the DRG are also engaged in neuropathic pain induced by paclitaxel, including $\mathrm{p}-\mathrm{PKC}, \mathrm{p}-\mathrm{NF}-\kappa \mathrm{B}, \mathrm{PDE} 4 \mathrm{D}$, and MCP-1; and tempol reduced pain behaviors by decreasing the levels of those mediators (Kim et al. 2017). Consistent with these findings, in the current study we observed that paclitaxel amplified IL-1 $\beta$, IL-6 and TNF- $\alpha$ in the DRG. Interestingly, systemic administration of tempol, vitamin C or GKT137831 attenuated increases of IL-1 $\beta$, IL- 6 and TNF- $\alpha$ in the DRG of paclitaxel rats whereas they attenuated mechanical and thermal hypersensitivity. Results of the present report suggest that IL-1 $\beta$, IL-6 and TNF- $\alpha$ in the DRG together with ROS contribute to mechanical pain and thermal hypersensitivity in paclitaxel-induced neuropathy.

In this report, paclitaxel was made in $40 \%$ DMSO and the same vehicle solution was used in control animals (Chen et al. 2011) and then we compared differences in pain responses between control rats and rats with paclitaxel. Paclitaxel induced neuropathic pain as compared with controls. However, it should be noted that DMSO was reported to lead to apoptotic degeneration in the central nerve system (Hanslick et al. 2009). Thus, we can't rule out the side effects of DMSO used in the current study. Nonetheless, a combination solution (i.e. cremophor EL/ethanol/saline) should be considered to make paclitaxel.

\section{Conclusions}

In the DRG of paclitaxel rats, Nrf2-ARE signal and SOD expression are downregulated and oxidative products and PICs are amplified, leading to mechanical pain and thermal hypersensitivity. Antioxidation or NOX inhibition by tempol/vitamin C and GKT137831 has a significant inhibitory effect on neuropathic pain induced by paclitaxel, accompanied with decreasing of PICs in the DRG. Nonetheless, our data reveal abnormal signaling pathways leading to mechanical pain and thermal hypersensitivity evoked by paclitaxel, including impairment of Nrf2-ARE and SOD, and enhancement of NOX4. Thus, balancing of ROS by administration of free radical scavengers and/or antioxidants and prevention of ROS generation appear significant to yield beneficial effects in neuropathic pain conditions after application of chemotherapeutic paclitaxel.

\section{Conflict of Interest}

There is no conflict of interest.

\section{Acknowledgements}

We thank all the people who helped during performing all the experiments. Hua Miao and Jinrong Xu had equal contributions to this work as co-first authors; and Xiaoming Zhao and Li Liu are corresponding authors. 


\section{References}

ALTENHOFER S, KLEIKERS PW, RADERMACHER KA, SCHEURER P, ROB HERMANS JJ, SCHIFFERS P, HO H, WINGLER K, SCHMIDT HH: The NOX toolbox: validating the role of NADPH oxidases in physiology and disease. Cell Mol Life Sci 69: 2327-2343, 2012.

ALTENHOFER S, RADERMACHER KA, KLEIKERS PW, WINGLER K, SCHMIDT HH: Evolution of NADPH oxidase inhibitors: selectivity and mechanisms for target engagement. Antioxid Redox Signal 23: 406-427, 2015.

CHAPLAN SR, BACH FW, POGREL JW, CHUNG JM, YAKSH TL: Quantitative assessment of tactile allodynia in the rat paw. J Neurosci Methods 53: 55-63, 1994.

CHEN Y, YANG C, WANG ZJ: Proteinase-activated receptor 2 sensitizes transient receptor potential vanilloid 1, transient receptor potential vanilloid 4 , and transient receptor potential ankyrin 1 in paclitaxel-induced neuropathic pain. Neurosci 193: 440-451, 2011.

DOUGHERTY PM, CATA JP, CORDELLA JV, BURTON A, WENG HR: Taxol-induced sensory disturbance is characterized by preferential impairment of myelinated fiber function in cancer patients. Pain 109: 132-142, 2004.

DUGGETT NA, GRIFFITHS LA, MCKENNA OE, DE SANTIS V, YONGSANGUANCHAI N, MOKORI EB, FLATTERS SJ: Oxidative stress in the development, maintenance and resolution of paclitaxel-induced painful neuropathy. Neurosci 333: 13-26, 2016.

FIDANBOYLU M, GRIFFITHS LA, FLATTERS SJ: Global inhibition of reactive oxygen species (ROS) inhibits paclitaxel-induced painful peripheral neuropathy. PloS One 6: e25212, 2011.

GAVAZZI G, BANFI B, DEFFERT C, FIETTE L, SCHAPPI M, HERRMANN F, KRAUSE KH: Decreased blood pressure in NOX1-deficient mice. FEBS Lett 580: 497-504, 2006.

GHERSI D, WILLSON ML, CHAN MM, SIMES J, DONOGHUE E, WILCKEN N: Taxane-containing regimens for metastatic breast cancer. Cochrane Database Syst Rev 6: CD003366, 2015.

GOLD R, KAPPOS L, ARNOLD DL, BAR-OR A, GIOVANNONI G, SELMAJ K, TORNATORE C, SWEETSER MT, YANG M, SHEIKH SI, DAWSON KT: Placebo-controlled phase 3 study of oral BG-12 for relapsing multiple sclerosis. New Eng J Med 367: 1098-1107, 2012.

HANNA M, ZYLICZ Z: Cancer Pain. Springer, London, 2013, 286 p.

HANSLICK JL, LAU K, NOGUCHI KK, OLNEY JW, ZORUMSKI CF, MENNERICK S, FARBER NB: Dimethyl sulfoxide (DMSO) produces widespread apoptosis in the developing central nervous system. Neurobiol Dis $\mathbf{3 4}$ : $1-10,2009$.

HOSKIN PJ: Radiotherapy. In: Clinical Pain Management: Cancer Pain. SYKES N, BENNETT MI, YUAN C-S (eds), Hodder Arnold, London, 2008, pp 251-255.

JAGGI AS, SINGH N: Therapeutic targets for the management of peripheral nerve injury-induced neuropathic pain. CNS Neurol Disord Drug Targets 10: 589-609, 2011.

JANES K, DOYLE T, BRYANT L, ESPOSITO E, CUZZOCREA S, RYERSE J, BENNETT GJ, SALVEMINI D: Bioenergetic deficits in peripheral nerve sensory axons during chemotherapy-induced neuropathic pain resulting from peroxynitrite-mediated post-translational nitration of mitochondrial superoxide dismutase. Pain 154: 2432-2440, 2013.

KALLENBORN-GERHARDT $\mathrm{W}$, SCHRODER K, DEL TURCO D, LU R, KYNAST K, KOSOWSKI J, NIEDERBERGER E, SHAH AM, BRANDES RP, GEISSLINGER G, SCHMIDTKO A: NADPH oxidase-4 maintains neuropathic pain after peripheral nerve injury. J Neurosci 32: 10136-10145, 2012.

KALLENBORN-GERHARDT W, SCHRODER K, GEISSLINGER G, SCHMIDTKO A: NOXious signaling in pain processing. Pharmacol Ther 137: 309-317, 2013.

KIM HK, HWANG SH, ABDI S: Tempol ameliorates and prevents mechanical hyperalgesia in a rat model of chemotherapy-induced neuropathic pain. Front Pharmacol 7: 532, 2017.

KIM HK, HWANG SH, LEE SO, KIM SH, ABDI S: Pentoxifylline ameliorates mechanical hyperalgesia in a rat model of chemotherapy-induced neuropathic pain. Pain Physician 19: E589-E600, 2016. 
KIM JH, DOUGHERTY PM, ABDI S: Basic science and clinical management of painful and non-painful chemotherapy-related neuropathy. Gynecol Oncol 136: 453-459, 2015.

LAM GY, HUANG J, BRUMELL JH: The many roles of NOX2 NADPH oxidase-derived ROS in immunity. Semin Immunopathol 32: 415-430, 2010.

MOI P, CHAN K, ASUNIS I, CAO A, KAN YW: Isolation of NF-E2-related factor 2 (Nrf2), a NF-E2-like basic leucine zipper transcriptional activator that binds to the tandem NF-E2/AP1 repeat of the beta-globin locus control region. Proc Natl Acad Sci U S A 91: 9926-9930, 1994.

PASETTO LM, D’ANDREA MR, ROSSI E, MONFARDINI S: Oxaliplatin-related neurotoxicity: How and why? Crit Rev Oncol Hematol 59: 159-168, 2006.

PORTENOY RK: Treatment of cancer pain. Lancet 377: 2236-2247, 2011.

SALVEMINI D, LITTLE JW, DOYLE T, NEUMANN WL: Roles of reactive oxygen and nitrogen species in pain. Free Radic Biol Med 51: 951-966, 2011.

SERETNY M, CURRIE GL, SENA ES, RAMNARINE S, GRANT R, MACLEOD MR, COLVIN LA, FALLON M: Incidence, prevalence, and predictors of chemotherapy-induced peripheral neuropathy: A systematic review and meta-analysis. Pain 155: 2461-2470, 2014.

SUZUKI Y, HATTORI K, HAMANAKA J, MURASE T, EGASHIRA Y, MISHIRO K, ISHIGURO M, TSURUMA K, HIROSE Y, TANAKA H, YOSHIMURA S, SHIMAZAWA M, INAGAKI N, NAGASAWA H, IWAMA T, HARA H: Pharmacological inhibition of TLR4-NOX4 signal protects against neuronal death in transient focal ischemia. Sci Rep 2: 896, 2012.

TANABE Y, HASHIMOTO K, SHIMIZU C, HIRAKAWA A, HARANO K, YUNOKAWA M, YONEMORI K, KATSUMATA N, TAMURA K, ANDO M, KINOSHITA T, FUJIWARA Y: Paclitaxel-induced peripheral neuropathy in patients receiving adjuvant chemotherapy for breast cancer. Int J Clin Oncol 18: 132-138, 2013. 